\title{
Autonomous microfluidic transport using electrowetting-based valves and integrated cells
}

\author{
Papaorn Siribunbandal, ${ }^{1}$ Shigeki Yamaguchi, ${ }^{2}$ Kenichi Kojima, ${ }^{2}$ Junji Fukuda, ${ }^{2}$ and \\ Hiroaki Suzuki ${ }^{2, a)}$ \\ ${ }_{1}^{1}$ Department of Physics, Faculty of Science and Technology, Thammasat University, 99 Moo 18, Klong 1, \\ Klong Luang, Pathumthani 12121, Thailand \\ ${ }^{2}$ Graduate School of Pure and Applied Sciences, University of Tsukuba, 1-1-1 Tennodai, Tsukuba, \\ Ibaraki 305-8573, Japan
}

(Received 21 July 2009; accepted 25 October 2009; published online 19 November 2009)

\begin{abstract}
Autonomous control of the transport of solutions in microfabricated flow channels using electrowetting-based valves and integrated electrochemical cells is proposed. An electrowetting-based valve formed with a gold electrode was opened when an electrolyte solution reached a zinc electrode in a controlling flow channel and a potential was applied to the gold electrode, causing the transport of the solution in the flow channel. Independent composite electrodes consisting of gold and zinc could function in the same manner without requiring additional electrodes. Controlled autonomous transport of solutions in a network of flow channels could also be carried out using the integrated valves. (C) 2009 American Institute of Physics. [doi:10.1063/1.3263956]
\end{abstract}

Next-generation microfluidic devices are expected to find applications in the autonomous operation of integrated components. This type of operation has conventionally been realized by driving the components using electrical signals provided by an external instrument. Ideally, however, these components should operate according to the instructions programmed in the chip, as in the case of electronic devices. To realize the same for microfluidic transport, electrowetting provides a realistic solution. Electrowetting is a phenomenon in which the wettability of a metal surface or dielectric on an electrode changes in response to a change in the applied potential. $^{1,2}$ This phenomenon has mostly been used to construct so-called digital microfluidic devices that can handle droplets on electrodes covered with a hydrophobic insulating layer. $^{3-6}$ Although this phenomenon has been successfully employed in some devices, a voltage of some tens of volts or higher was applied in previous cases, and this restricted further applications. On the other hand, when the solution is in direct contact with the electrode, a change can be induced by a small voltage $(\sim 1 \mathrm{~V})$. The ability to couple the electrode with other electrochemical components has already led to the development of promising devices. ${ }^{7-9}$ In the latter approach, it may be possible to integrate electrochemical cells in a flow channel to drive the components and also activate the cells directly when an electrolyte solution reaches the electrodes of the cell. In other words, the instructions for switching the components may potentially be programmed in the shapes and dimensions of the flow channel network.

To demonstrate this concept, we initially used the test device shown in Fig. 1(a) (type I). This device is made of glass (Corning, \#7740) and poly(dimethylsiloxane) (PDMS) (Shin-Etsu Chemical, KE-1300T). Two parallel flow channels with injection ports and open ends were formed with PDMS by replica molding using patterns of a thick-film

\footnotetext{
a) Author to whom correspondence should be addressed. Tel.: +8129-853-5598. FAX: +81-29-853-4490. Electronic mail: hsuzuki@ims.tsukuba.ac.jp.
}

photoresist (MicroChem, SU-8) as a template. The width and depth of each flow channel were 500 and $40 \mu \mathrm{m}$, respectively. In each flow channel, gold electrodes (500 $\times 500 \mu \mathrm{m}^{2}$ ) were formed as valves, and zinc electrodes $\left(500 \times 500 \mu \mathrm{m}^{2}\right)$ were formed as the cells. The valve re-

(a)

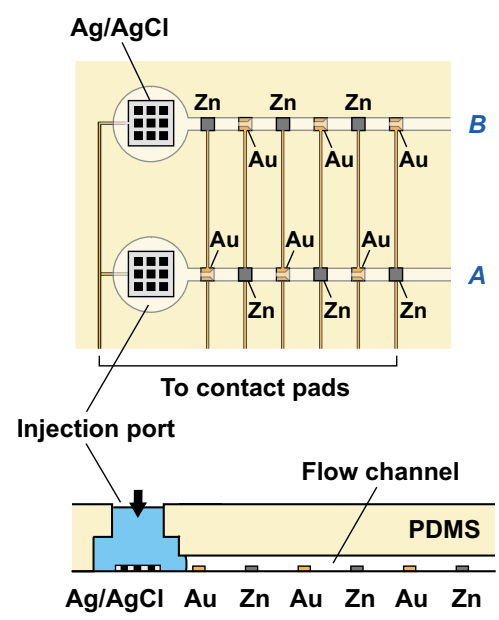

(b)

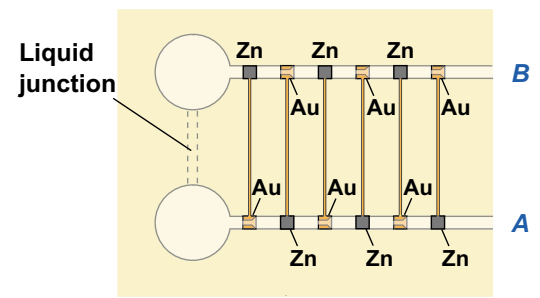

FIG. 1. (Color online) Microfluidic devices used for characterization. (a) Type I device with gold and zinc electrodes along with two $\mathrm{Ag} / \mathrm{AgCl}$ electrodes. The lower figure shows the cross-section along flow channel A. The leads extending to the bottom are for the measurement of the generated voltage. (b) Type II and III devices with gold and zinc electrodes. In the type II device, the flow channels are connected with a liquid junction (dashed line) at the injection ports, whereas they are separated in the type III device. 
gions including the gold electrodes were constricted to a width of $200 \mu \mathrm{m}$ to block the solution more effectively before the cell was activated. ${ }^{10}$ The electrodes were located alternately within and between the flow channels and they were connected to form six pairs. One of the zinc electrodes and the $\mathrm{Ag} / \mathrm{AgCl}$ electrodes formed cells. To explore the possibility of constructing more advanced systems, we also used devices without the $\mathrm{Ag} / \mathrm{AgCl}$ electrodes [Fig. 1(b)]. In one of these devices, the flow channels were connected at the injection ports with a liquid junction (type II). In the other, the flow channels were separated (type III). The valve electrodes and the base layer for the zinc and $\mathrm{Ag} / \mathrm{AgCl}$ electrodes were formed using a thin-film gold/chromium layer. Zinc was electroplated onto the base layer by applying a current of $50 \mu \mathrm{A}$ for $20 \mathrm{~min}$ in a stirred solution containing $1.8 \mathrm{M}$ $\mathrm{ZnCl}_{2}$ and $4.9 \mathrm{M} \mathrm{NH}_{4} \mathrm{Cl}$ at $35^{\circ} \mathrm{C}$. A fluorescein solution containing $0.1 \mathrm{M} \mathrm{KCl}$ was used to visualize the movement of liquid columns.

In our previous studies, we had used a valve structure surrounded by a gold electrode and three walls made of PDMS. ${ }^{10}$ In this structure, a solution stopped initially and started to move upon the application of a voltage of approximately -0.6 to $-0.7 \mathrm{~V}$ (versus $\mathrm{Ag} / \mathrm{AgCl}$ ). Considering this, we first used a combination of zinc and $\mathrm{Ag} / \mathrm{AgCl}$ for each cell in the type I device. The electrodes exhibit characteristic potentials based on the following reactions: $\mathrm{Zn}^{2+}+2 \mathrm{e}^{-} \rightleftarrows \mathrm{Zn}$; $\mathrm{AgCl}+\mathrm{e}^{-} \rightleftarrows \mathrm{Ag}+\mathrm{Cl}^{-}$. Zinc ions were not added to the solution in order to make the potential of the zinc electrode as negative as possible. The open-circuit voltage of the cell was $0.91 \mathrm{~V}(n=5)$, which was sufficient to trigger the passage of the solution through the valve. Figure 2 shows the movement of the solutions. When the first solution was injected into flow channel A, it stopped at the first valve [Fig. 2(a)]. When another solution was then injected into flow channel $\mathrm{B}$, it passed the first zinc electrode and stopped at the first valve. While the solution passed the zinc electrode, the voltage generated between the zinc and $\mathrm{Ag} / \mathrm{AgCl}$ electrodes was applied between the valve electrode and the $\mathrm{Ag} / \mathrm{AgCl}$ electrode in flow channel A. Following this, the solution passed the valve area and moved to the second valve in flow channel A [Fig. 2(b)]. Thereafter, the steps were repeated and columns in the two flow channels stopped and moved forward alternatively [Figs. 2(c) and 2(d)].

The change that occurs at the zinc electrode/solution interface should have an influence on the polarizable gold electrode connected to the zinc electrode. Therefore, the gold electrode may be charged up and polarized without the Ag/ $\mathrm{AgCl}$ electrodes. ${ }^{11,12}$ To verify this, we used the type II and III devices. First, we filled the flow channels with a $0.1 \mathrm{M}$ $\mathrm{KCl}$ solution. Then, the potential of one of the gold electrodes was measured with respect to a $\mathrm{Ag} / \mathrm{AgCl}$ wire electrode inserted in the corresponding injection port. The potential was stable and was $-0.75 \mathrm{~V}(n=5)$ and $-0.64 \mathrm{~V}(n$ $=5$ ) for the type II and type III devices, respectively. In the type II device, solutions moved as in the case of the type I device. In the type III device, however, solutions occasionally stopped at some valves, which may be due to the more positive potential near the threshold. Even with this device, the potential of the gold electrode shifted to the negative direction by approximately $60 \mathrm{mV}$ when the size of the zinc electrode was tripled, and the solutions moved more smoothly than expected. Alternatively, the application of a
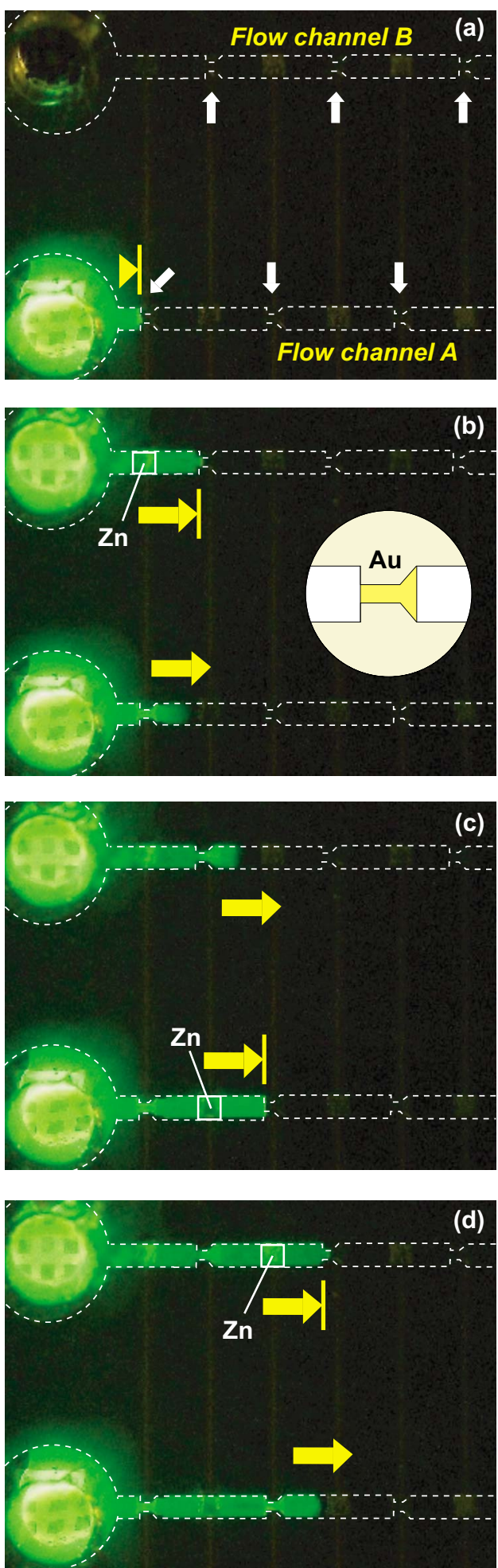

FIG. 2. (Color online) Fluorescence images showing the movement of fluorescein solutions in the type I device. The inset of (b) shows a magnified view of the valve area. The small arrows indicate the valves, whereas the large arrows indicate the movement of the solutions. The vertical short bars on the right-hand side of the large arrows indicate that the liquid column was stopped at the corresponding valves.

microscopically rough gold surface shifts the threshold to the positive direction and will have a similar effect. ${ }^{13}$

More complicated movement of solutions can be realized by forming more valves in the type II and III devices. Figure 3(a) shows a device with a controlling flow channel and flow channels A-D with injection ports connected to 
(a)
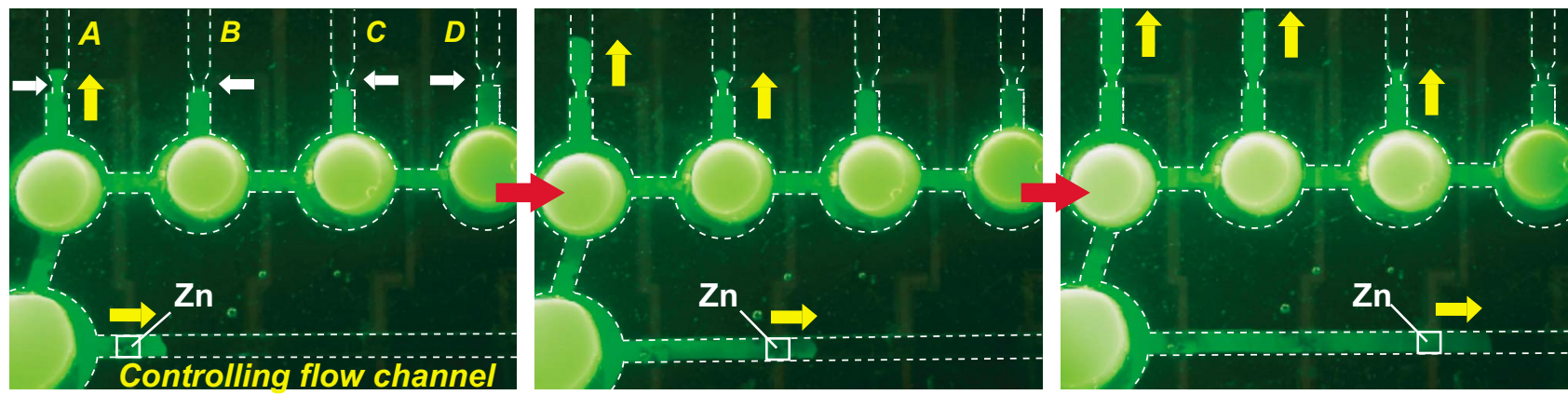

(b)
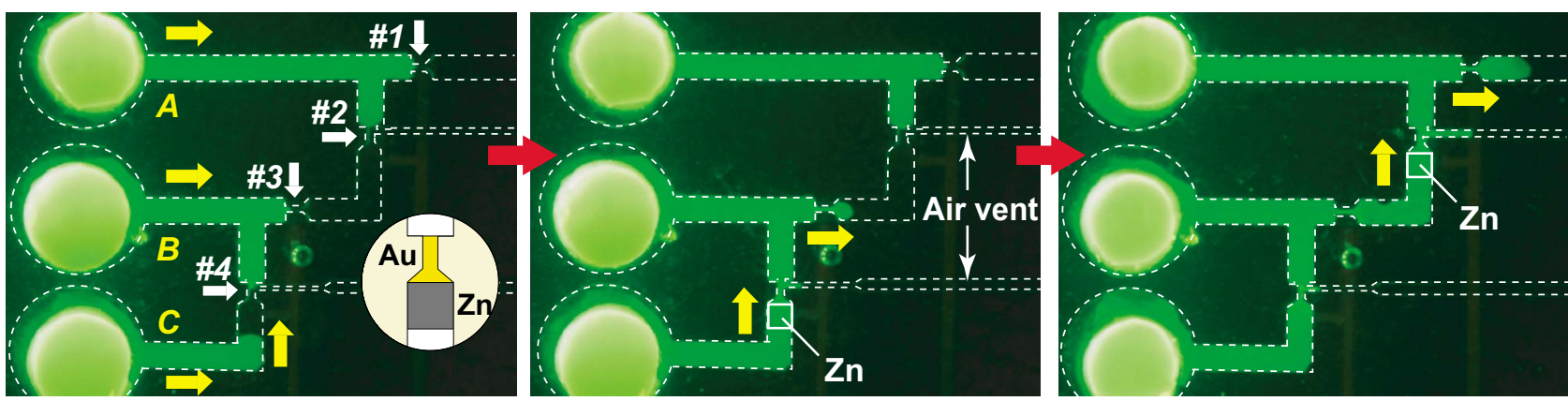

FIG. 3. (Color online) Devices for more complicated microfluidic transport. (a) Device with four flow channels and a controlling flow channel. (b) Device with four valves in a network of flow channels. Valves 2 and 4 consist of gold and zinc (inset of the leftmost picture). The structures of the valves in (a) and valves 1 and 3 in (b) are the same as those of the ones shown in Fig. 2. The small arrows indicate the valves, whereas the large arrows indicate the movement of the solutions.

each other. Valves were formed at the injection ports and they were connected to the zinc electrodes in the controlling flow channel. The injection ports were connected to the liquid junctions. When solutions were injected into flow channels A-D, they stopped at the valves. However, when another solution was injected into the controlling flow channel, the valves opened one-by-one beginning with the one in flow channel A when the solution in the controlling flow channel passed the zinc electrodes. Figure 3(b) shows a device with flow channels A-C. Valves 1 and 3 were the same as those in the previous devices. On the other hand, valves 2 and 4 consisted of composite electrodes consisting of gold and zinc that existed in the same flow channel (see the inset). Valves 1 and 2 were connected with a gold lead. Valves 3 and 4 were also connected with a gold lead. When solutions were introduced into flow channels A and B, they stopped at valves 1, 2 , and 3. Then, another solution was injected into flow channel C. When the solution wetted the zinc area of valve 4, the solution moved onto the gold electrode and the two solutions on both sides of the valve merged. At the same time, valve 3 connected with the zinc area of valve 4 opened. When the solution injected from valve 3 reached valve 2 , it opened in a manner similar to valve 4 , and at the same time, valve 1 connected with the zinc area of valve 2 opened. Note that the movement of the solutions through valves 2 and 4 is unidirectional.

To conclude, autonomous microfluidic transport can be realized using electrowetting-based valves and integrated cells. Independent composite electrodes consisting of gold and zinc could be used to control the transport of solutions without requiring additional electrodes. Controlled valve action can be achieved using a properly designed network of flow channels and electrodes.

This study was partially supported by a Grant-in-Aid for Scientific Research (B) (Grant No. 19360369) from the Japan Society for the Promotion of Science.

${ }^{1}$ C. Quilliet and B. Berge, Curr. Opin. Colloid Interface Sci. 6, 34 (2001). ${ }^{2}$ F. Mugele and J.-C. Baret, J. Phys.: Condens. Matter 17, R705 (2005).

${ }^{3}$ J. Lee, H. Moon, J. Fowler, T. Schoellhammer, and C.-J. Kim, Sens. Actuators A 95, 259 (2002).

${ }^{4}$ M. G. Pollack, A. D. Shenderov, and R. B. Fair, Lab Chip 2, 96 (2002).

${ }^{5}$ V. Srinivasan, V. K. Pamula, and R. B. Fair, Lab Chip 4, 310 (2004).

${ }^{6}$ M. Abdelgawad and A. Wheeler, Adv. Mater. 21, 920 (2009).

${ }^{7}$ N. Nashida, W. Satoh, J. Fukuda, and H. Suzuki, Biosens. Bioelectron. 22, 3167 (2007)

${ }^{8}$ H. Hosono, W. Satoh, M. Toya, K. Morimoto, J. Fukuda, and H. Suzuki, Sens. Actuators B 132, 614 (2008).

${ }^{9}$ S. Yamaguchi, K. Morimoto, J. Fukuda, and H. Suzuki, Biosens. Bioelectron. 24, 2171 (2009).

${ }^{10}$ W. Satoh, H. Yokomaku, H. Hosono, N. Ohnishi, and H. Suzuki, J. Appl. Phys. 103, 034903 (2008).

${ }^{11}$ A. J. Bard and L. R. Faulkner, Electrochemical Methods: Fundamentals and Applications (Wiley, New York, 1980).

${ }^{12}$ J. O. M. Bockris, A. K. N. Reddy, and M. Gamboa-Aldeco, Modern Electrochemistry (Kluwer Academic/Plenum, New York, 2000), Vol. 2A.

${ }^{13}$ H. Yokomaku, W. Satoh, J. Fukuda, and H. Suzuki, J. Appl. Phys. 104, 064910 (2008). 